livraisons

d'Histoire

de l'Architecture

\section{Livraisons de l'histoire de l'architecture}

33 | 2017

Histoire du (des ?) patrimoine(s)

\title{
Restauration des monuments historiques versus production nouvelle : l'Encyclopédie d'architecture et la gazette des architectes et du bâtiment (1851-1869)
}

Restauration of architectural heritage versus new production: the

Encyclopédie d'architecture and the Gazette des architectes et du bâtiment (1851-1869)

Restaurierung von Denkmalen versus Neuproduktion. Die 'Encyclopédie d'architecture' und die 'Gazette des architectes du bâtiment' (1851-1869)

Bérénice Gaussuin

\section{(2) OpenEdition}

Journals

Édition électronique

URL : http://journals.openedition.org/lha/713

DOI : $10.4000 /$ lha. 713

ISSN : 1960-5994

Éditeur

Association Livraisons d'histoire de l'architecture - LHA

Édition imprimée

Date de publication : 15 juin 2017

Pagination : 19-32

ISSN : $1627-4970$

\section{Référence électronique}

Bérénice Gaussuin, «Restauration des monuments historiques versus production nouvelle I'Encyclopédie d'architecture et la gazette des architectes et du bâtiment (1851-1869) ", Livraisons de I'histoire de l'architecture [En ligne], 33 | 2017, mis en ligne le 16 juin 2019, consulté le 15 octobre 2019 URL : http://journals.openedition.org//ha/713; DOI : 10.4000//ha.713 


\section{RESTAURATION DES MONUMENTS HISTORIQUES VERSUS PRODUCTION NOUVELLE : L'ENCYCLOPÉDIE D'ARCHITECTURE ET LA GAZETTE DES ARCHITECTES ET DU BÂTIMENT (1851-1869)}

«Voir c'est savoir ${ }^{1}$."

La restauration des édifices anciens et la création architecturale semblent aujourd'hui deux champs d'une même discipline qui peinent à communiquer entre eux. Pour comprendre cet état actuel, le retour à ce qui apparaît comme sa genèse est nécessaire. Le XIX siècle invente un nouveau champ d'action pour l'architecte. La définition du terme "Restauration " évolue également avec cet élargissement des compétences et donne lieu à un débat entre les différents penseurs de ce siècle. Viollet-le-Duc formule sa théorie de la restauration dans le tome VIII du Dictionnaire raisonné ${ }^{2}$, publié en 1866 . Le terme existe déjà auparavant et est très utilisé dans la section architecture de l'École des Beaux-Arts puisque les «restaurations " sont les projets qui font suite aux relevés des antiques et qui, accompagnés d'un mémoire, constituent les envois des pensionnaires de la Villa Médicis à Rome ${ }^{3}$. Ce sont des projets qui ne sont pas destinés à être réalisés, comme des reconstitutions en tant qu'exercice intellectuel. L'usage de ce mot avec ce sens va continuer pendant tout le XIX ${ }^{\mathrm{e}}$ siècle et remplace d'ailleurs celui de "restitution " utilisé jusqu’à la fin du XVIII ${ }^{e}$ siècle $^{4}$.

C'est à travers deux revues que nous tenterons de décrypter les évolutions de la théorie de l'architecture : l'Encyclopédie d'architecture et la Gazette des architectes et du bâtiment qui, comme l'indique le titre de la thèse de Béatrice Bouvier, sont "un miroir de l'architecture de [leur] temps ${ }^{5} \%$. Les éditeurs et directeurs de publication ${ }^{6}$,

1. Eugène Viollet-le-Duc, Histoire d'un dessinateur. Comment on apprend à dessiner, J. Hetzel \& $\mathrm{C}^{\mathrm{ie}}$, Paris, 1879, p. 302.

2. Eugène Viollet-le-Duc, Dictionnaire raisonné de l'architecture française du XI au XVI siècle (10 tomes), B. Bance et A. Morel, Paris, 1854-1868.

3. Pierre Pinon, François-Xavier Amprimoz, Les Envois de Rome (1778-1968). Architecture et archéologie, École française de Rome, Palais Farnèse, 1988.

4. Ibid., p. 25.

5. Béatrice Bouvier, L'Encyclopédie d'architecture (1850-1892) : un miroir de l'architecture de son temps, thèse de doctorat sous la direction de Jean-Michel Leniaud, EPHE, Paris, 1999.

6. Le choix de ces deux revues est motivé également par le fait qu'elles sont très liées au milieu qui porte cet engagement envers les monuments historiques. Cf. : Béatrice Bouvier, L'Édition d'architecture à Paris au XIX siècle. Les maisons Bance et Morel et la presse architecturale, Droz, Genève, 2004. 
affirment des positionnements par les choix éditoriaux de ces deux revues qui mêlent les sujets de la construction nouvelle et des monuments historiques. Seront également repérés le vocabulaire, les mots employés, qu'il s'agisse d'articles sur les monuments historiques ou sur une construction nouvelle, de façon à déterminer l'influence de la constitution progressive des doctrines d'intervention sur les monuments avec en tête de file Eugène Viollet-le-Duc.

\section{Deux revues, une ligne éditoriale}

L'Encyclopédie et la Gazette ${ }^{7}$ sont deux revues liées : la seconde ${ }^{8}$ remplace la première à partir de 1863 . Cependant, le choix du titre de la revue reflète une évolution de sa vision. Alors que le terme " encyclopédie " propose une compilation de tous les savoirs d'une discipline, "l'architecture ", la "gazette " se situe dans un propos de proximité, de quotidienneté. Alors que la première formule est dans une distanciation toute scientifique et une ambition encyclopédique, la seconde cherche une efficience, un contact direct avec le terrain pour mieux faire passer ses messages.

Le choix des illustrations placées en frontispice de ces revues reflète leur programme. L'Encyclopédie propose une accumulation de monuments de tous temps et tous horizons, illustrant la vision encyclopédique de sa mission' ${ }^{9}$ (ill. 1). La Gazette propose un chantier en cours, en pleine activité, avec un grand nombre de personnages en train de travailler $^{10}$ (ill. 2). Ici, la baraque de chantier fume. Partout, des échafaudages, des engins de levage, les pierres sont suspendues dans le vide. Au pied de l'édifice, on les pousse, on les tire pour les déposer au bas de l'échafaudage sur lequel elles se hisseront bientôt pour rejoindre leur assise et compléter le puzzle de la construction/restauration en cours. Cette image vivante est une métaphore du programme de la revue qui veut être dans une actualité permanente dans l'action et proche des praticiens, de ceux qui construisent, qui sont sur le chantier.

Pour servir ce dessein, la Gazette fait un choix différent quant à la place des illustrations ${ }^{11}$. L'Encyclopédie publiait de grandes planches de dessin d'architecture géométral. La Gazette n'abandonne pas ce langage spécifique à l'architecte et aux acteurs du bâtiment tout en lui ajoutant, dans le texte, des illustrations utilisant

7. Béatrice Bouvier, "Répertoire des périodiques d'architecture", Les Périodiques d'architecture. $X V I I I^{e}-X X^{e}$ siècle. Recherche d'une méthode critique d'analyse, Jean-Michel Leniaud et Béatrice Bouvier (dir.), École des chartes, Paris, 2001.

8. La Gazette des architectes et du bâtiment naît du regroupement de l'Encyclopédie et de la Gazette du bâtiment qui était un complément à la Revue Générale de l'architecture. Cf. Marc Saboya, Presse et architecture au XIX siècle. César Daly et la Revue générale de l'architecture et des travaux publiques, Picard, Paris, 1991.

9. L'Encyclopédie ne propose un frontispice qu'à partir de sa deuxième année, 1852.

10. Béatrice Bouvier, L'Édition d'architecture à Paris au XIX siècle, op. cit. Edmond Corroyer, auteur du dessin, choisit un temple grec, pas une cathédrale gothique (p. 81).

11. Béatrice Bouvier, "Une illustration didactique, scientifique et professionnelle ", L'Édition d'architecture au XIX siècle, op. cit., p. 83-114. 


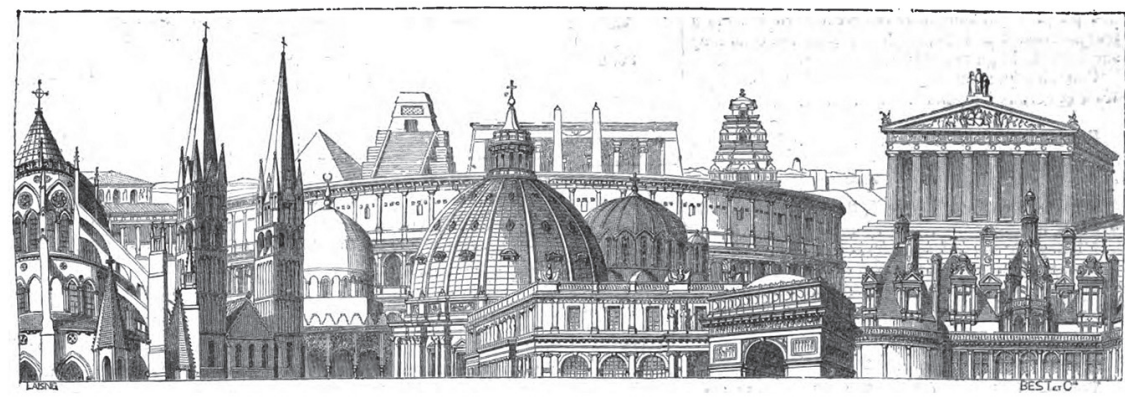

Ill. 1 : Frontispice de l'Encyclopédie d'architecture, $1^{\mathrm{er}}$ mars 1852 (première publication avec un frontispice, il restera le même tout au long de la publication). (Source : extrait d'une numérisation Google, programme Google Recherche de livre).

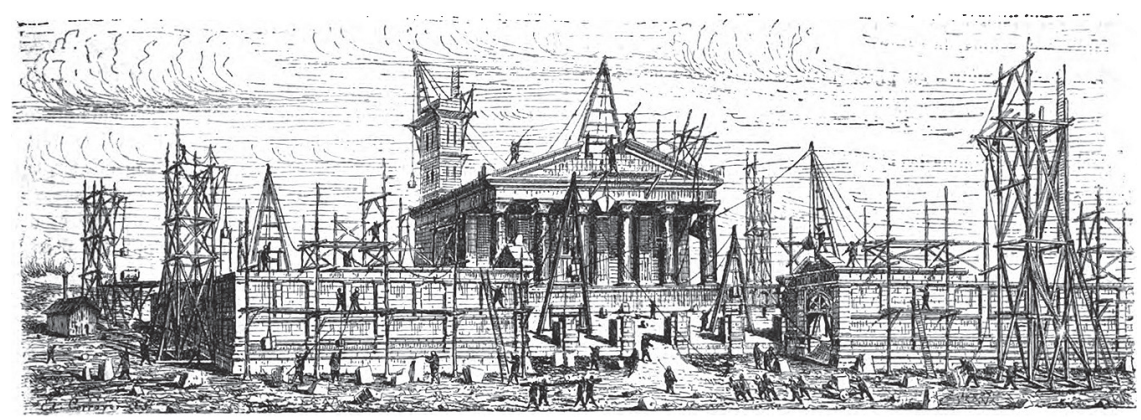

Ill. 2 : Edmond Corroyer, frontispice de la Gazette des architectes et du bâtiment, $\mathrm{n}^{\circ}$ 3, 1863. (Source : extrait d'une numérisation Google, programme Google Recherche de livre).

l'axonométrie et la perspective, plus directement appréhendables. Les praticiens se voient faciliter la compréhension des nouveaux procédés.

Finalement, l'Encyclopédie, dès sa deuxième année de publication change de ligne éditoriale. Tout en gardant à l'esprit son objectif encyclopédique qui aboutira à la publication d'édifices dans les moindres détails, elle s'attache très tôt à ce que continuera ensuite la Gazette : la proximité avec les praticiens. Ce deuxième objectif empêchant l'exhaustivité encyclopédique, la Gazette, bien que se plaçant sous le chapeau de l'Encyclopédie d'architecture, l'abandonne en conscience ${ }^{12}$.

\section{Construction neuve et restauration : des notions mêlées}

Dès la première année, l'Encyclopédie publie conjointement les dessins d'édifices aussi divers que la cathédrale Notre-Dame de Paris, le comble en fer de l'Hôtel de

12. La revue ne se prêtant pas de la manière la plus aisée au travail de l'encyclopédiste, ce travail de compilation est laissé à des ouvrages de synthèse. D'ailleurs, l'Encyclopédie annonce, dès 1853, soit un an avant le début de sa publication, le Dictionnaire raisonné de Viollet-le-Duc. 
ville de Paris, les plans de niveaux de la Bibliothèque Sainte-Geneviève et les dessins préalables à la "réparation " du Pont-Neuf, y compris des dessins d'installation de chantier. Les approches, les propositions, les époques sont variées. Ces édifices font l'objet d'articles dès la deuxième année explicitant le parti du concepteur. Le comble en fer des magasins des Forges de la Providence ${ }^{13}$ qui figure sur 5 planches durant la première année, est traité en feuilleton d'articles ${ }^{14}$ l'année suivante. Ils évoquent avec une grande précision les problématiques de résistance des matériaux liées à ces innovations techniques et leurs coûts. La revue permet la diffusion de nouveaux procédés qui s'ajoutent à la boîte à outils des praticiens, l'objectif à atteindre étant de fournir de bons exemples de mises en œuvre d'innovations, pour qu'ils servent de références aux architectes. L'actualité technique est donc présente, aussi bien dans les dessins que dans le texte. La pratique de la restauration se retrouve notamment à travers la publication épisodique des dessins du Pont-Neuf à Paris. 9 planches sont publiées au cours de la première année parmi lesquelles figurent aussi bien des relevés des existants que des dessins des " cintres pour l'abaissement des voûtes ${ }^{15}$ ". Ces dessins sont ceux que l'architecte fournit à l'entrepreneur pour l'exécution de ces étais et pourraient être utilisés par d'autres sur d'autres chantiers.

La Bibliothèque Sainte-Geneviève d'Henri Labrouste est un bâtiment qui mobilise l'éditeur pendant les dix premières années de la publication. La première année, seule une planche ${ }^{16}$ représentant les plans de niveaux de l'édifice est publiée. La deuxième année, 17 planches sont publiées, la troisième année, 19 planches, une planche la quatrième année, 2 planches la cinquième et 5 planches la septième année. Soit un total de 45 planches comprenant des dessins généraux et de détails permettant une vision complète de l'édifice comme l'annonce le programme ${ }^{17}$ de la revue dès 1851 .

Un édifice équivalent dans la quantité de documents qui sont publiés est la cathédrale Notre-Dame de Paris qui fait l'objet de la publication de 43 planches entre la première et la septième année ${ }^{18}$. Cet édifice est publié dans sa totalité pour montrer le travail des architectes restaurateurs ${ }^{19}$, au même titre que celui du concepteur de la Bibliothèque Sainte-Geneviève.

Ces exemples illustrent la publication conjointe et sans distinction d'innovations techniques, de travaux de restauration d'un ouvrage d'art, d'une conception architecturale novatrice et d'un chantier phare de restauration. Mais l'Encyclopédie

13. Dépôt de fers ou magasins des Forges de la Providence, quai Jemmapes à Paris, par l'architecte Léonide Marquet.

14. "Ferronnerie. Fers spéciaux des Forges de la Providence et comble en fer des magasins de cet établissement ", Encyclopédie d'architecture, no III du $1^{\text {er }}$ janvier 1852, p. 25-28. Le deuxième article de la série est publié dans le $\mathrm{n}^{\circ} \mathrm{V}$ du $1^{\mathrm{er}}$ mars 1852, p. 33-37.

15. Encyclopédie d'architecture, 1851, planche $\mathrm{n}^{\text {os }} 19$ et 20, 4 planches seront publiées la deuxième année.

16. Ibid., planche $\mathrm{n}^{\circ} 73$.

17. Victor Calliat, Encyclopédie d'architecture, première année, Bance, Paris, 1851.

18. Les planches qui concernent la sacristie de Notre-Dame de Paris sont exclues car c'est un édifice à part entière qui nécessite un traitement spécifique.

19. Pour Notre-Dame de Paris, Jean-Baptiste Lassus et Eugène Viollet-le-Duc. 
publie également une grande variété d'articles qui ne font pas partie de son programme initial. Aux choix éditoriaux de la première année s'ajoutent des actualités diverses ${ }^{20}$ comme annoncées dans le préalable à la deuxième année ${ }^{21}$. Le passé et le présent sont représentés à égalité, comme équivalents. Le dialogue entre les innovations et les découvertes archéologiques est proposé aux lecteurs assidus de la revue.

À partir de 1863 la Gazette prend le relais. L'examen des "tables des sommaires » année par année montre l'étiolement progressif de la part de la restauration des monuments par rapport à celle des constructions nouvelles. La revue ne revendiquant plus le passé mais uniquement le présent, dès le premier numéro de 1863, aucune restauration n'est évoquée. Les procédés techniques, qui sont nombreux à être exposés dans la revue, peuvent à la fois servir à la restauration d'édifices existants et à de nouvelles constructions ${ }^{22}$. Au numéro quatre de la même année, l'éditeur publie un article sur le château de Pierrefonds, une restauration certes, mais il n'évoque que la "Charpente en fer du corps de logis principal du château de Pierrefonds ${ }^{23}$ ". De ce travail de Viollet-le-Duc sur le château, seule la partie d'innovation est publiée. C'est donc ce qui prime pour la Gazette : l'innovation, y compris dans les monuments historiques qui ne se suffisent plus à eux-mêmes. La part des restaurations pour elles-mêmes rattrape quelque peu son retard à partir du $\mathrm{n}^{\circ} 11$ de la même année avec la publication de la restauration du château de Saint-Germain.

Les tables annuelles et les thématiques qu'elles choisissent reflètent l'idée énoncée dans la profession de foi de l'Encyclopédie. Le passé et le présent sont mêlés et doivent être pris par le praticien au même niveau. La "table générale " de la première année ${ }^{24}$ distingue les programmes - architecture religieuse ou civile - puis les détails sont classés par corps de métier : charpente, serrurerie, mosaïque. Ces tables évoluent au fil des publications, s'enrichissant d'autres termes, mais en gardant des approches thématiques semblables à la première. La pratique de la restauration et celle de la construction nouvelle y sont mélangées : la "table analytique et alphabétique » ${ }^{25}$ de l'année 1863 de la Gazette fait figurer dans la catégorie "Construction ", la restauration de Notre-Dame de Paris et la construction de la manufacture impériale de Sèvres. En 1865, les deux pratiques ne sont toujours pas différenciées, la dichotomie entre restauration et création ne semble pas exister. La part de la restauration s'est néanmoins considérablement réduite : sur 25 édifices dans la catégorie "Architecture », seulement 5 sont présentés comme des restaurations. Le déséquilibre apparaît clairement, la nouveauté prend le dessus. Dans la table de $1866^{26}$, un seul article porte le mot : "Restauration ": il est relégué à la catégorie "Divers ".

20. Le sommaire du $\mathrm{n}^{\circ} \mathrm{X}$ du $1^{\text {er }}$ août 1852 se présente comme suit : "Salon 1852, distribution des récompenses. - Concours sur la théorie des voûtes. - Cirque d'hiver, par M. Hittorf, architecte [...]. »

21. "Avis de l'éditeur ", Encyclopédie d'architecture, deuxième année, Bance, Paris, 1852, p. 1-3.

22. Par exemple : "La plomberie repoussée ", Gazette des architectes et du bâtiment, n 2, 1863, p. 45.

23. "Charpentes en fer. Charpente en fer du corps de logis principal du château de Pierrefonds ", Gazette des architectes et du bâtiment, no 4, 1863, p. 44-46.

24. Encyclopédie d'architecture, 1851, p. V-XIII.

25. Gazette des architectes et du bâtiment, 1863, p. 335.

26. Gazette des architectes et du bâtiment, 1866, p. 388. 
L'étude du passé reste cependant bien présente : l'archéologie est toujours en bonne place dans les publications. Elle apparaît généralement dans la catégorie "Études artistiques, archéologiques et scientifiques ${ }^{27}$ " qui présente pêle-mêle, en 1868-1869, les découvertes faites récemment à Rome par M. Devrez, architecte, ainsi qu'une étude sur les églises contemporaines d'Anatole de Baudot et un nouveau traité de perspective par David Sutter. Les mêmes architectes publient sur des sujets divers. Ainsi, en 1866, Félix Narjoux présente à la fois une étude archéologique du baptistère de Valence ${ }^{28}$ et l'abattoir qu'il construit à Nice ${ }^{29}$.

Ces choix éditoriaux dessinent une vision simultanée de la restauration et de la création en architecture mais la part de la restauration s'amenuise progressivement. L'approche de la restauration est nouvelle, elle est en train de se délimiter peu à peu, mais dans le champ et avec les outils et les mots de l'architecte car c'est lui qui la prend en charge, dans sa discipline.

\section{Les mots de la construction nouvelle/les mots des monuments historiques}

Chaque année, l'Encyclopédie, puis la Gazette, publient un long rapport sur l'exposition d'architecture du Salon. Ces articles et les mots qu'ils emploient ${ }^{30}$ sont révélateurs de la vision simultanée des deux approches de la discipline. Les termes utilisés sont clairement identifiés : "étude" désigne les études au sens où on emploie le terme de nos jours. Le mot "projet " concerne la conception en tant que telle, le moment où l'architecte projette, imagine ce qu'il va faire et le traduit par une production de dessins, de maquettes. Le terme "construction nouvelle " est employé pour les projets réalisés, construits.

L'usage du mot "projet ", à des fins polémiques, dans un article concernant la construction de l'Opéra de Paris ${ }^{31}$ publié en 1863, démontre cette répartition entre les termes :

«La réalisation [...] d'un projet d'école [...] que l'on croyait irréalisable, [...] jusqu'à ce jour était destiné à paraître quelques heures dans les salles d'exposition du palais des Beaux-Arts, pour aller s'enfouir, après obtention d'une grande médaille, dans les cartons de son auteur ${ }^{32}$."

27. Gazette des architectes et du bâtiment, 1868-1869, p. 266-267.

28. Félix Narjoux, "Baptistère de Valence. Étude archéologique ", Gazette des architectes et du bâtiment, 1866, p. 225-226.

29. Félix Narjoux, "De la construction d'un abattoir ", Gazette des architectes et du bâtiment, 1866, p. 33-35.

30. Alice Thomine, "Les tables des matières des revues d'architecture (1850-1900)», Jean-Michel Leniaud et Béatrice Bouvier (dir.), Les Périodiques d'architecture, op. cit., p. 136-146. L'auteure, à partir de l'analyse des occurrences de certains types d'édifices, ne souhaite pas conclure à la prédominance des idées rationalistes dans les revues qui nous concernent mais préfere parler d'illustration de "l'état de la commande architecturale au XIX" siècle".

31. "Le nouvel Opéra ", Gazette des architectes et du bâtiment, n 3, 1868, p. 29-30.

32. Ibid., p. 29. 
"Projet» est un terme péjoratif, critique et exprime le sentiment de l'auteur: ce projet aurait dû rester de papier et ne jamais être construit ! Dans l'ensemble de l'article, on dénombre 7 occurrences du mot " projet » dont 5 désignent directement l'Opéra de Charles Garnier en cours d'exécution ${ }^{33}$ contre une occurrence du terme " construction ", au début, pour signaler que la chose est en cours. L'utilisation du terme "réalisation" à la ligne suivante semble souligner le fait que ce "projet d'école [...] irréalisable" se matérialise bel et bien dans la réalité construite de la ville de Paris. Le projet est même qualifié par le terme "d'école " ${ }^{34}$ pour ajouter au dénigrement de ce travail qui relève donc des concours de l'École des Beaux-Arts et de ses "grandes médailles " ${ }^{35}$. Deux occurrences du mot "projet " ne se rapportent pas à celui de Garnier. Elles désignent une autre proposition exposée lors du concours, restée dans son statut de projet car destinée à rejoindre les "cartons de son auteur " et, à ce titre, bien plus intéressante. L'auteur conclut son article : "C'est là un projet de grand prix, avec ses défauts, ses impossibilités, son absence de sens pratique, et ses qualités dues à une jeune et brillante imagination. »

Le vocabulaire des interventions dans les monuments historiques est plus flou. C'est celui de la discipline de l'architecte, enrichi de celui de l'archéologue avec des termes tels que "relevés " ou "état actuel ». Le compte rendu de l'exposition d'architecture du salon de 1869 permet de le clarifier ${ }^{36}$ : "Nous classerons [...] les œuvres exposées en quatre groupes $[\ldots]: 1^{\circ}$ les dessins, $2^{\circ}$ les restaurations, $3^{\circ}$ les projets, $4^{\circ}$ les édifices. "

La catégorisation de Lawrence Chapron nous permet de comprendre les usages, car il les définit :

« $1^{\circ}$ Dessins. - [...] Un dessin d'architecture n'est pas seulement un relevé purement géométrique, $[. .$.$] un document historique, [...] une aquarelle [...].$ Un dessin d'architecture [...] doit être le résultat d'une étude complète de l'objet qu'il est destiné à représenter ${ }^{37}$."

La représentation par le dessin doit être si complète qu'elle permet la compréhension et la connaissance de l'édifice sans même avoir vu ou expérimenté l'espace. L'auteur définit ensuite la restauration :

« $2^{\circ}$ Restaurations. [...] travaux [nécessitant] [...] l'esprit d'observation et de déduction indépendamment de toutes les autres qualités que doit aussi posséder l'architecte. C'est $[. .$.$] à cette condition qu'on peut arriver à restituer$

33. Une première inauguration a lieu en 1867 , mais l'édifice n'est pas entièrement achevé.

34. En italique dans le texte original.

35. C'est à se demander qui a écrit cet article qui n'est pas signé : on connaît la bataille de Violletle-Duc contre les Beaux-Arts et son enseignement et on sait également que le même a perdu le concours qu'il pensait gagner pour la construction de cet opéra.

36. Lawrence Chapron, "Salon de 1869. Architecture ", Gazette des architectes et du bâtiment, n 17 , 1868-1869, p. 161-166.

37. Ibid., p. 161. 
ou à conserver aux édifices leur véritable caractère, à exécuter ces travaux dans des circonstances délicates, sans mettre les constructions en péril $[\ldots]^{38}$."

Dans cette partie de l'article, le terme " projet » est accolé, une seule fois, à " de restauration ». L'auteur parle plus volontiers de "la restauration" seule à laquelle s'ajoute "l'agrandissement " (une occurrence ${ }^{39}$ ), "véritable transformation " ${ }^{40}$ (une occurrence). "Additions" est également utilisée dans le même contexte. On note deux occurrences de "projet " pour parler d' "agrandissement et remaniement du château de Martinvast (Manche) par M. White» jugés très négativement par l'auteur. Est-ce pour cela que l'auteur lui refuse le terme de restauration? Il l'utilise pourtant à onze reprises dans cette section, désignant des approches différentes : il caractérise les cas confrontant l'architecte à un édifice ancien. Le constat est celui d'une grande confusion dans les termes et leurs usages alors que pour les autres catégories le vocabulaire est fixé, clair. En témoignent la troisième et la quatrième catégories : " projets" et « édifices» (ill. 3).

\begin{tabular}{|c|c|c|c|c|c|}
\hline & Termes utilisés & $1^{\circ}$ Dessin & $2^{\circ}$ Restauration & $3^{\circ}$ Projet & $4^{\circ}$ Edifices \\
\hline \multirow{5}{*}{$\begin{array}{c}\text { Constructions } \\
\text { nouvelles }\end{array}$} & Projet & 0 & 2 & 29 & 3 \\
\hline & Conception & 0 & 0 & 0 & 5 \\
\hline & Composition & 1 & 0 & 18 & 2 \\
\hline & Exécution/exécuter & 0 & 1 & 0 & 11 \\
\hline & Construction/construire & 4 & $1+2$ Reconstruction & 5 & 9 \\
\hline \multirow{2}{*}{$\begin{array}{l}\text { Monuments } \\
\text { historiques }\end{array}$} & Restauration & 2 & 12 & 0 & 0 \\
\hline & Relevé & 9 & 0 & 0 & 0 \\
\hline
\end{tabular}

Ill. 3 : Tableau récapitulatif des occurrences des termes repérés dans l'article "Salon de 1869 " par Lawrence Chapron, Gazette des architectes et du bâtiment, n 17, 1868-1869, pp. 161-166. (C) B. Gaussuin.

Cette approche par les mots révèle que le vocabulaire de ce que nous appelons aujourd'hui la création ${ }^{41}$ est très défini et permet de désigner des objets sans aucune confusion. Aujourd'hui le terme de projet est utilisé de manière plus floue : la frontière entre le construit et le papier est plus trouble puisque l'architecte parle de ses projets réalisés pour ce que le XIX ${ }^{e}$ siècle appelle construction ou simplement édifice. Le vocabulaire du neuf est plus riche et plus précis que celui de la restauration ; ce dernier terme restant un "fourre-tout». Aujourd'hui, un grand nombre d'autres mots tels que "réhabilitation, transformation, rénovation..." ayant chacun un sens différent, qualifie les approches ${ }^{42}$ de l'existant, la restauration ${ }^{43}$ étant réservée aux monuments historiques.

38. Ibid., p. 162.

39. Ibid., p. 162, à propos de l'église Saint-Saturnin (Oise) par M. Rouseau.

40. Ibid., p. 163, à propos de la restauration du château de Montbrison dans le Tarn-et-Garonne, par M. Trilhe.

41. Par opposition à la restauration.

42. Dominique Rouillard, Architectures contemporaines et monuments historiques: guide des réalisations en France depuis 1980, Éd. Le Moniteur, Paris, 2006.

43. L'usage actuel de ce terme est connoté : est-on du côté de l'architecture ou du côté des monuments historiques? 


\section{Nouvelle pratique, nouveaux savoirs}

Cette nouvelle discipline, dont le vocabulaire est en train de se constituer, nécessite également une approche nouvelle des objets. L'architecte doit avoir une nouvelle attitude face à l'existant. Françoise Choay l'indique de la façon suivante :

"Les études préparatoires à la conservation et à la restauration des monuments historiques ont demandé l'acquisition supplémentaire de nombreux savoirs scientifiques et techniques nouveaux ${ }^{44}$."

L'Encyclopédie puis la Gazette vont prendre à bras le corps cette tâche nouvelle, car les architectes qui sont à l'œuvre sur les monuments historiques ne sont plus dans les écoles mais déjà sur le terrain, face aux édifices. Ces publications s'adressent à eux. Elles publient des relevés, des articles techniques, des actualités pratiques et des exemples de bonnes réalisations. Parfois, au détour d'un article du dernier type, l'auteur énonce une méthode plus générale: l'approche méthodologique est en train de se constituer. Dans un article consacré à la reprise en sous-œuvre de l'église de Saint-Benoît-sur-Loire ${ }^{45}$, on s'attend à un exposé détaillé et très technique de la manière dont l'architecte a mené son chantier. L'auteur, Anatole de Baudot, va livrer cet exposé mais donner également une stratégie d'intervention :

"Les architectes chargés de ce genre de travaux ont dû, indépendamment de la question purement artistique, faire face aux difficultés pratiques les plus sérieuses, et $[. .$.$] ils ont su généralement mener à bonne fin les reprises les$ plus difficiles."

L'approche des monuments historiques présente donc des spécificités qui s'ajoutent à celles de la discipline de l'architecte. Il doit désormais résoudre des problèmes techniques bien distincts de ses prérogatives habituelles : les « questions purement artistiques ". Pour Baudot, il ne doit pas y avoir de recette, il faut tout réinventer à chaque édifice :

«De l'étude des divers travaux de restauration faits jusqu’à ce jour vouloir déduire une méthode générale, quant aux modes d'étaiements, est impossible, parce que deux cas semblables ne se présentent pour ainsi dire jamais $[\ldots]^{46}$. "

C'est pour cela qu'il faut être architecte pour restaurer un édifice. Parce qu'il faut inventer des solutions propres à chaque situation et seul l'architecte est capable d'une telle créativité. Ainsi, les étaiements ne peuvent pas faire l'objet de copie, il faut à chaque fois en inventer de nouveaux.

44. Françoise Choay, Allégorie du Patrimoine, Seuil, Paris, 1992, p. 113.

45. Anatole de Baudot, "Église de Saint-Benoît-sur-Loire. Restauration. Reprise en sous-œuvre des piliers de la crypte. M. Lisch, architecte ", Gazette des architectes et du bâtiment, 1865, p. 209-212.

46. Ibid., p. 209. 
«Mais c'est [...] fournir d'utiles renseignements que de publier [...] des opérations de reprise les plus intéressantes [...], cette publication n'aurait-elle pour résultat que de faire voir [...] combien il importe à quiconque entreprend la restauration d'un édifice d'en comprendre l'esprit, [...] le principe général de construction, combien il importe [...] que la direction de semblables travaux soit prise par l'architecte lui-même $[\ldots]^{47}$."

La méthode est donnée. D’abord, connaître l'édifice, dans ses moindres détails, dans son fonctionnement, maîtriser son histoire qui permettra à l'architecte d'inventer les solutions pour le restaurer. Donc la Gazette donne de bons exemples, non pour les copier mais, comme pour les constructions nouvelles, pour nourrir les architectes d'un système de références. À travers l'exposé de cette méthode, l'auteur va plus loin : si elle s'applique aux monuments historiques, elle doit également être celle de la construction nouvelle. Il suffit de remplacer "restauration " par « construction » et l'enseignement d'Eugène Viollet-le-Duc est là. Baudot parle de restauration et de création sur le même plan, ce sont des notions qui ne sont pas traitées différemment par les architectes du XIX ${ }^{\mathrm{e}}$ siècle qui se saisissent de l'histoire.

\section{Deux revues, une doctrine}

Ce mélange des genres s'explique par la pensée dont la revue est porteuse : celle de Viollet-le-Duc. Dans les débuts de la revue il n'apparaît que très peu, ou en filigrane. À partir de 1866, il est progressivement plus présent. Son nom apparaît de manière significative, en particulier pour ses constructions neuves. Dans la table de 1866, c'est l'église paroissiale de Saint-Denis qui occupe le plus de place dans la section "architecture ». Sa pensée théorique est également de plus en plus clairement énoncée.

En 1865, le constat évoqué plus haut réapparaît : une nouvelle fois, pour la restauration de Pierrefonds, c'est un comble en fer qui est le sujet de l'article signé A.B. (Anatole de Baudot ?) :

"Quoique ce comble présente une disposition générale assez compliquée [...], sa combinaison générale comme ses détails sont d'une extrême simplicité. Cet exemple montre [...] quels services peut rendre le fer dans l'établissement des combles, combien il se prête rationnellement à toutes les exigences $[\ldots]^{48}$. "

Cet article sur la pratique dans les édifices anciens est un plaidoyer pour un matériau innovant. Les restaurations de monuments historiques doivent, au même titre que les constructions neuves, porter l'innovation, l'inventivité, la créativité propre à l'architecte.

47. Ibid.

48. "Château de Pierrefonds. Restauration. Comble en fer", Gazette des architectes et du bâtiment, 1865 , p. 280. 
L'année suivante, c'est une conférence de Viollet-le-Duc à la Sorbonne qui est publiée dans son intégralité. Elle s'intitule "De l'architecture dans ses rapports à l'histoire ${ }^{49}$ " : «L'architecture dans ses rapports avec l'histoire !... Il existe donc des rapports entre l'histoire et l'architecture? La réponse ne peut être qu'affirmative ${ }^{50}$. "

Après un long développement dans lequel il s'attache à démontrer que la grande architecture est celle qui répond à la civilisation dans laquelle elle est construite, il conclut :

"L'architecture, pour mériter le nom d'art, doit être l'expression exacte des besoins, des goûts, des aptitudes d'une civilisation. Mais, quand pour se produire, au lieu de recourir aux sources du vrai, elle va butinant partout des idées étrangères à son temps, à son pays, à la nature des populations, à leurs aptitudes, alors ce n'est plus un art mais la plus dispendieuse de toutes les fantaisies humaines ${ }^{51}$."

La messe est dite. L'histoire permet la production d'œuvres en parfaite adéquation avec leur temps, c'est le projet de Viollet-le-Duc pour le sien. L'histoire et l'archéologie ne sont que des moyens, ils ne sont pas des fins mais des outils pour penser l'architecture "moderne ${ }^{52}$ ".

Alors que la doctrine est clairement énoncée par la voix forte d'Eugène Violletle-Duc, elle s'exprime aussi dans des lieux inattendus : une série d'articles de son fils, Eugène-Louis, sur la restauration du château de Saint-Germain ${ }^{53}$, paraît dans la Gazette dès la première année. Dans un long développement, publié en feuilleton, l'auteur reprend d'abord la démarche à adopter : "Il était impossible de comprendre ses anciennes dispositions, à moins de les avoir spécialement étudiées ${ }^{54}$. "

L'architecte doit d'abord bien connaitre l'édifice dont il a la charge pour mieux l'appréhender. L'auteur expose ensuite le projet d'Eugène Millet, lauréat du concours organisé pour l'occasion. Les travaux de restauration sont exposés au fur et à mesure de leur exécution. Plusieurs articles ${ }^{55}$ traitent du château sur des points de détails techniques et il faut attendre le numéro $15^{56}$ pour que l'auteur révèle enfin l'idée sous-jacente à la publication longue et précise de ce travail : alors que les démolitions permettent de comprendre les dispositions anciennes du château, l'auteur se dévoile.

49. Eugène Viollet-le-Duc, "De l'architecture dans ses rapports à l'histoire : conférence à la Sorbonne ", Gazette des architectes et du bâtiment, n 23,1866 , p. 353-364.

50. Ibid., p. 353.

51. Ibid., p. 364 .

52. On emploie ici le terme "moderne" au sens où le XIX ${ }^{\mathrm{e}}$ siècle l'utilise c'est-à-dire lié à son temps.

53. Eugène Viollet-le-Duc (fils), "Château de Saint-Germain (Restauration) ", Gazette des architectes et du bâtiment, $\mathrm{n}^{\circ} 11,1863$, p. 141-148.

54. Ibid., p. 141.

55. Eugène Viollet-le-Duc (fils), "Charpente. Plafond de l'une des salles du donjon du Château de Saint-Germain ", Gazette des architectes et du bâtiment, no 14, 1863, p. 186-188.

56. Eugène Viollet-le-Duc (fils), "Restauration du château de Saint-Germain ", Gazette des architectes et du bâtiment, $\mathrm{n}^{\circ} 15,1863$, p. 197-204. 


\begin{abstract}
«Dans presque tout ce qui se fait de nos jours comme architecture, il est impossible de trouver un enseignement, soit que la construction montre un désaccord complet entre les formes adoptées et les destinations, soit qu'elle accuse une incompatibilité [...] entre la nature des matériaux employés et la façon dont ils ont été mis en œuvre. [...] il faut chercher longtemps avant de rencontrer un édifice dont l'extérieur soit [...] l'expression d'un besoin et dont la structure soit basée sur la nature des matériaux. L'architecture est de tous les arts celui qui doit emprunter le plus au raisonnement [...]. Cette vérité $[\ldots]$ est peu reconnue de notre temps où le raisonnement est généralement sacrifié à la fantaisie. [...] Lorsque les formes d'une architecture sont raisonnées, elles ne font jamais naître l'idée de les remplacer par d'autres ; elles rendraient d'ailleurs cette substitution impossible ${ }^{57}$."
\end{abstract}

La voix du père résonne auprès du fils qui note assidûment. Ainsi est-on fixé sur la doctrine et sur la ligne éditoriale de la Gazette, étonnamment dans le long exposé en plusieurs épisodes de la restauration d'un édifice, et non dans l'analyse d'un bâtiment neuf ou dans une tribune clairement affichée comme telle. Finalement, c'est la valeur de l'exemple qui compte le plus et la démonstration qui en résulte est implacable. L'enseignement par l'observation des œuvres du passé est l'affaire du chantier mené par Millet. Dans l'article du numéro suivant, l'auteur opère de nouveau ce passage de la pratique à la théorie. La partie est le tout et le tout est la partie ${ }^{58}$ : "Ce ne sera jamais [...] une masse de matériaux empilés les uns sur les autres sans accuser de structure ; ce sera toujours un assemblage raisonné et dont chaque partie aura non seulement une valeur absolue, mais encore une valeur relative impossible à nier ${ }^{59}$."

La théorie s'expose à travers la pratique expliquant pourquoi la place des restaurations diminue avec les années: seuls les bons exemples, ceux capables de porter la théorie, sont publiés. Car la Gazette, dans les choix de ses contenus, montre bien qu'elle fait sienne cette pensée martelée par Viollet-le-Duc dans ses Entretiens sur l'architecture ${ }^{60}$.

$\mathrm{Au}$ fur et à mesure que se forme la doctrine de la restauration et le corps d'architectes et d'inspecteurs qui la pratiquent, ce nouveau champ disciplinaire, très présent au début de la publication des revues étudiées, disparaît peu à peu. La doctrine portée par Viollet-le-Duc, quant à elle, est de plus en plus présente et plus particulièrement développée dans les publications et les choix éditoriaux de ces deux revues entre 1851 et 1869. L'influence de ses deux ouvrages majeurs publiés dans les

57. Ibid., p. 200-201.

58. Hubert Damisch, "Introduction ", L'Architecture raisonnée. Extraits du dictionnaire de l'architecture française réunis et présentés par Hubert Damisch, Hermann, Paris, 1964.

59. Eugène Viollet-le-Duc (fils), "Restauration du château de Saint-Germain ", Gazette des architectes et du bâtiment, $\mathrm{n}^{\circ} 16,1863$, p. 214-220.

60. Ces derniers sont le contenu des cours qu'il souhaitait professer à l'école des Beaux-Arts et sont publiés par son fils sous forme de résumés dans les colonnes de la Gazette. 
mêmes années - le Dictionnaire ${ }^{61}$ entre 1854 et 1868 et les Entretiens ${ }^{62}$ entre 1863 et 1872 - devient prépondérante. Dans le même temps, les restaurations disparaissent. La présence/absence des mots qui lui sont attachés dans les tables annuelles en témoigne, alors que la discipline de l'architecte garde ses mots avec leur usage précis et défini. Se pose alors la question suivante, à laquelle la recherche tâchera de répondre $^{63}$ : les restaurations à l'œuvre peuvent-elles encore illustrer la théorie et de la doctrine de Viollet-le-Duc ? Le cas du château de Saint-Germain longuement relaté dans les publications, légitime cette question, puisque les exemples développés permettront aux héritiers d'Eugène Viollet-le-Duc, de théoriser le rationalisme en architecture.

Bérénice GaUsSUIN architecte DESA et architecte du patrimoine, enseignante à l'ENSA Paris-Malaquais, doctorante

61. Eugène Viollet-le-Duc, Dictionnaire raisonné de l'architecture française du XI au XVI siècle (10 t.), B. Bance et A. Morel, Paris, 1854-1868.

62. Eugène Viollet-le-Duc, Entretiens sur l'architecture (2 vol.), A. Morel, Paris, 1863-1872.

63. La présente étude se prolongera pour apporter plus de précisions statistiques en incluant les publications de l'Encyclopédie d'architecture qui s'interrompent après les derniers numéros cités en 1869 et reprennent à partir de 1873 à l'initiative d'Eugène Viollet-le-Duc, reprenant son titre d'origine tout en incluant des aspects apportés par la Gazette. 
